12th International conference on Global Security, Safety and Sustainability (ICGS3-19)

\section{Introduction}

Social engineering (SE) attacks are a serious threat to online might subject people to different kinds of Despite increased concern with this risk, there has been little research activity focused upon social engineering in the potentially rich hunting ground of social networks. The number of victims of social engineering attacks will be decreased if the users' detection ability has improved. Yet, this improvement of the user's detection behaviour can't be occurred without investigating the users' weakness points. The present study develops a conceptual model to test the factors that influence social networks users' judgment of social engineering-based attacks in order to identify the weakest points of users' detection behaviour which also help to predict vulnerable individuals.

\section{Motivation and Background}

Security practitioners still relying on technical measures to protect from online threats while overlooking the fact that cybercriminals are targeting human weak points to spread and conduct their attacks (Krombholz et al, 2015). According to the human-factor report (2018), the number of using social engineering attacks to exploit human vulnerabilities has dramatically increased over the last year. This arises the necessity of finding a solution that helps the user to show acceptable defensive behaviour in the social network (SN) reality. Identifying the user characteristics that make them more or less vulnerable to social engineering threats is vital to protect against such threats. Identifying the weakest points can help users to recognise their perceptual and habitual limits and to target these limitations by a personalised advisory system that could be designed to fit the user needs which could provide new insights to social engineering mitigation solutions.

\title{
A Conceptual Model to Predict Social Engineering Victims
}

Samar Muslah Albladi ${ }^{1}$, George R S Weir

Department of Computer and Information Sciences

University of Strathclyde, UK

${ }^{1}$ samar.albladi@strath.ac.uk

3. Major Contributions
- Proposing a conceptual model that includes varieties of
user-related factors such as socio-psychological, habitual,
perceptual, and socio-emotional. Proposing such a novel
conceptual model helped in bridging the gap between
theory and practice by providing a better understanding of
how to predict vulnerable users based on different
perspectives of human-related factors.
- Proposing a new influencing perspective, socio-emotional,
that hasn't been satisfactory reported in the literature.
The current research aims to gain insight into user
competence in detecting security threats in the context of
online social networks and investigates the
multidimensional space that determines this user
competence level.

4. Methodology

Participants were presented with an online-questionnaire which incorporated three main parts:

- Demographic questions.

- Questions that measure the constructs of the proposed

- The scenario-based experiment which includes 6 images of Facebook posts (4 high-risk scenarios, and 2 low-risk scenarios). Each post contains a type of cyber-attack such as phishing, clickjacking, malware, phishing scam. Participants were asked to indicate their response to these attacks if they encounter them in their real accounts by answering number of questions.

- 316 participants have completed the study questionnaire. Participens' demographics include a variety of profiles in terms of gender ( $39 \%$ male, $61 \%$ female), education level, and major. However, the majority (76\%) of participass

\section{Experimental Design}

To evaluate the proposed model, partial least squares structural equation modelling (PLS-SEM) has been used due to its suitability of dealing with complex predictive models. SmartPLS v3 software package was used to analyse the model.

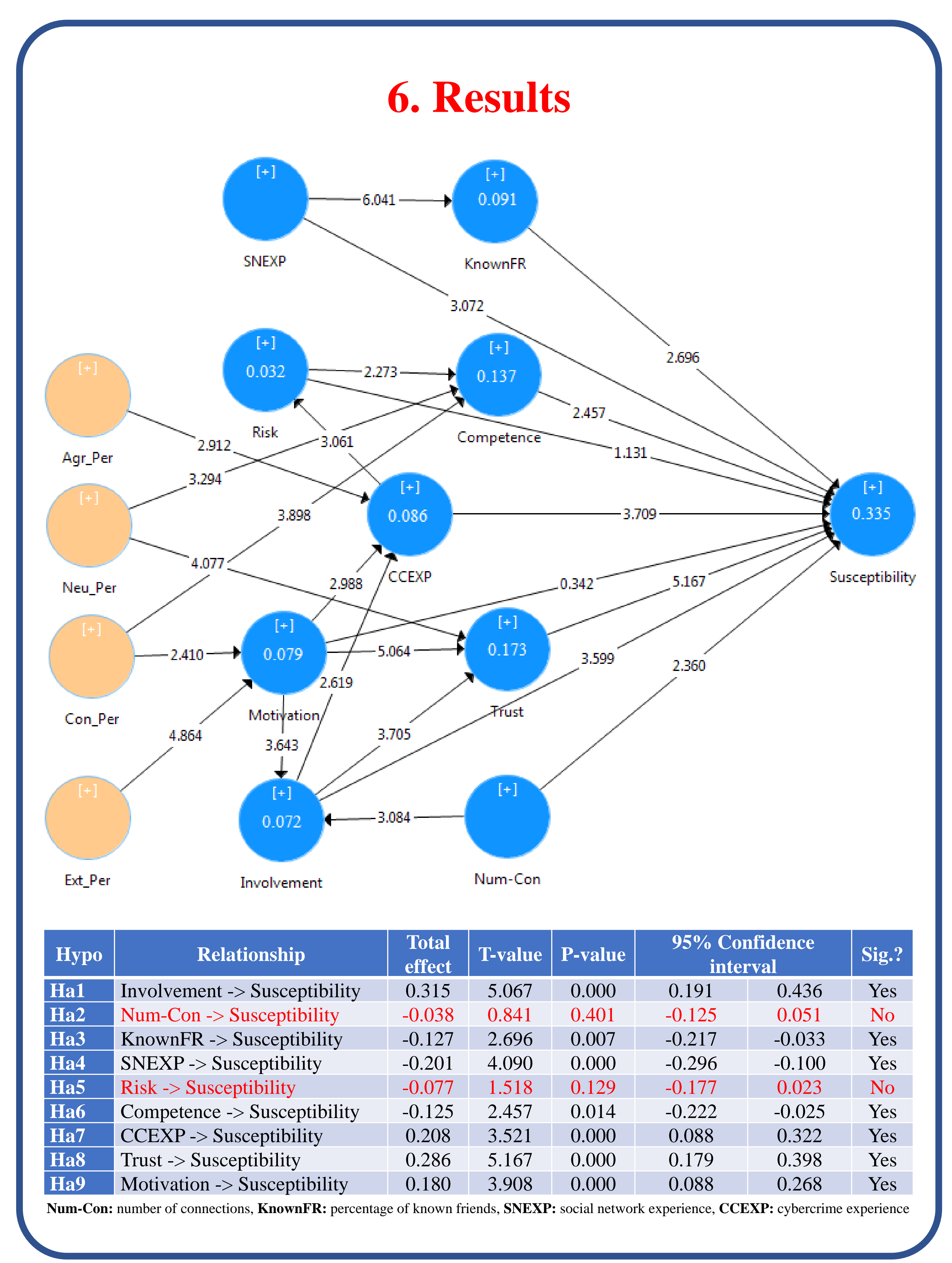

7. Discussion

The findings of this research indicate that most of the - Thsidered user characteristics factors influence users' vulnerability either directly or indirectly.

People trust in the social network's provider, and members were the strongest determinant of their vulnerability to SE attacks. This support our claims that socio-emotional factors are fundamental to consider.

The individual competence level to deal with cybercrime, which was measured based upon three dimensions, i.e., security awareness, privacy awareness, and self-efficacy, found to significantly predict the individual's ability to detect SE attacks on Facebook.

- The present study found personality traits (except openness to experience) to be significant predictors of human vulnerability to cyber-attacks. Yet, these relations are indirect and mediated by other relevant factors.

\section{Conclusions}

The present study provides evidence that individuals characteristics can predict vulnerable people to SE victimisation and their effects should be considered in future studies when designing training and awareness programs.

9. Future Work

This study on determining user vulnerabilities affords a basis for profiling users according to their characteristics and weakness in respect of particular threats.

In turn, this provides a means for future studies to design a personalised advisory system that sends awareness posts to target individual user needs. 